\title{
Probing the Concepts of Photonic Crystals on Scintillating Materials
}

\author{
Matthias Kronberger, Etiennette Auffray, Member, IEEE, and Paul Lecoq, Member, IEEE
}

\begin{abstract}
The high refractive index of current scintillating materials puts severe restrictions on their effective light yield. In this paper, we describe an approach that uses a photonic crystal pattern machined into the coupling face of the scintillator to partly overcome the problem of total internal reflection. Simulations are performed for $2 \mathrm{~mm} \times 2 \mathrm{~mm} \times 8 \mathrm{~mm}$ LuAP and LSO pixels with and without photonic crystal and different types of wrapping. It is shown that by tuning the structure of the photonic crystal and the size of its elements, the extraction efficiency of the surface can be significantly improved compared to a plain exit surface.
\end{abstract}

Index Terms-Light extraction, photonic crystals, scintillation detectors.

\section{INTRODUCTION}

$\mathbf{T}$ HE energy resolution of any organic or inorganic scintillator at a given energy $E$ is correlated via the photostatistics with the number of photons extracted from the crystal. This number depends not only on the number of photons produced when a particle interacts with the scintillating medium, but also on the light extraction efficiency (LEE) of the scintillator,

$$
N_{\gamma, \text { extracted }}=L E E \cdot N_{\gamma, \text { generated }} .
$$

This parameter depends on the absorption length of the scintillator at the wavelength of emission, its size, its geometrical shape, and the reflection and absorption properties of the enveloping material if a wrapping or coating is used to cover side and end faces. By adjusting these parameters, the LEE of the material can be improved and a higher number of photons can be extracted from the crystal. This increased light yield leads, in turn, to an improved energy resolution.

Another important limiting factor of the LEE is the transmission of the scintillator surface that is coupled with the photodetector. This is important especially for substrates with high refractive indices: In these cases, the angle of total reflection, $\theta_{\mathrm{tot}}$, will be relatively small and only a fraction of the generated photons will be able to leave the crystal. Therefore, finding a way to manipulate the transmission properties of the exit surface holds the potential to increase the total amount of light collected from the scintillating medium per particle event.

A possible solution to overcome the problem of total internal reflection was first discussed by [1], who suggested the usage of a thin photonic crystal (PhC) slab-a periodic pattern of two

Manuscript received June 28, 2007; revised December 3, 2007.

The authors are with CERN, 1211 Geneva 23, Switzerland (e-mail: matthias. kronberger@cern.ch; etiennette.auffray@cern.ch; paul.lecoq@cern.ch).

Digital Object Identifier 10.1109/TNS.2008.922827

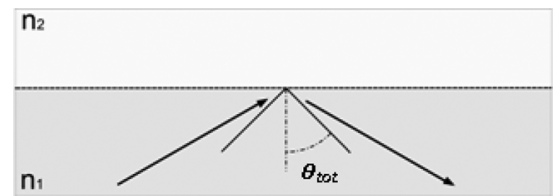

(a)

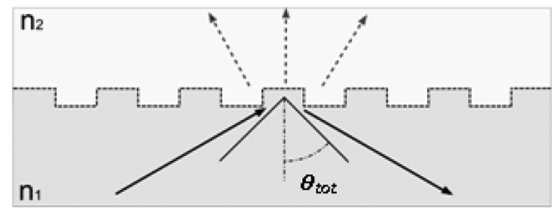

(b)

Fig. 1. Situation at the interface between two materials $\left(n_{1}>n_{2}\right)$ for (a) plain geometry and (b) a periodic modulation of the interface. In the case of plain geometry, any wave with incident angle $\theta>\theta_{\text {tot }}$ will be totally reflected. If a periodic modulation is apparent, several modes can be extracted from medium 1 and the transmission of the interface becomes $>0$.

materials with different dielectric constants-to diffract light outside the extraction cone from a high refractive index substrate. If an electromagnetic wave with wave vector $\vec{k}$ hits the interface between two media with $n_{1}>n_{2}$, the $z$ component of the wave vector in the medium above the substrate will be imaginary for $\theta>\theta_{\text {tot }}$ and no light will be transmitted through this interface (Fig. 1(a)). This changes if the interface comprises a periodic modulation (Fig. 1(b)): Since the dielectric constant $\varepsilon(\vec{r})$ of such a structure is periodic with any lattice vector $\vec{R}_{i}$ of the structure,

$$
\varepsilon(\vec{r})=\varepsilon\left(\vec{r}+\vec{R}_{i}\right), \quad i=1,2,
$$

the in-plane components $\vec{k}_{/ /}$of the wave vector are also periodic. In this case, the incoming wave is split into different harmonics and the $z$ component of the wave vector in medium 2 , $k_{z, n 2}^{p}$, becomes

$$
k_{z, n 2}^{p}=\sqrt{n_{2}^{2} \cdot k^{2}-\left(\vec{k}_{/ /}+\vec{G}_{j}\right)^{2}},
$$

where $\vec{G}_{j}$ is a reciprocal lattice vector,

$$
\vec{R}_{i} \cdot \vec{G}_{j}=2 \pi \delta_{i, j}
$$

If $k_{z, n 2}^{p}$ is real for one harmonic, this particular partial wave is diffracted out of the substrate and the light transmission of the interface is larger than zero. 


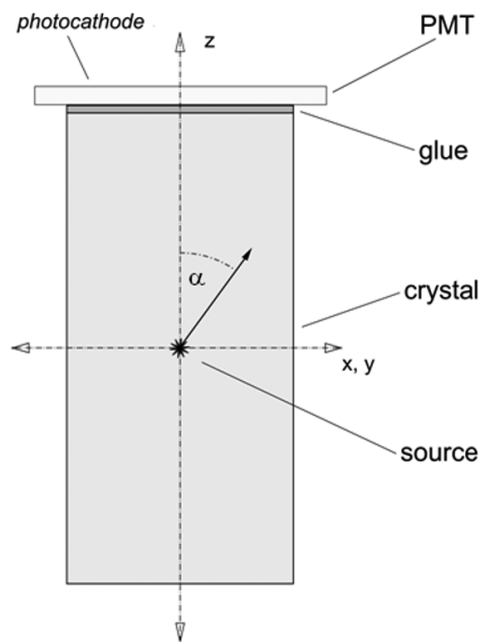

Fig. 2. Schematic view of the system simulated with LITRANI. The angle $\alpha$ corresponds to the angle between the $z$ axis and the $k$ vector of the photon. Except for the coupling face, all crystal faces are simulated either with a diffusing or a perfectly reflecting wrapping. The size of the crystal is $2 \mathrm{~mm} \times 2 \mathrm{~mm} \times 8$ $\mathrm{mm}$.

This approach has already been successfully used to improve light extraction from light-emitting diodes [2]-[8]. However, its potential to increase the LEE of scintillating materials with high refractive index has, to the best of our knowledge, not yet been studied. This work aims to assess some of the basic properties of such a system and its impact on the LEE.

\section{MATERIALS AND METHODS}

To evaluate the impact of a $\mathrm{PhC}$ pattern on the light extraction efficiency of scintillating materials with high refractive index, studies of the optical properties of scintillators with periodically modulated exit surface were performed and compared with the results for scintillators without modulation. This was necessary to do as the angular distribution of the photons that arrive at the exit surface was expected to differ from the angular distribution of all photons at the moment of generation. This effect is due to internal absorption of the scintillating light: photons that are emitted at larger angles relative to the $z$-axis have a longer path to travel before they reach the exit surface and therefore, are more likely to be absorbed by the scintillating material. This, in turn, has implications on the desired properties of the $\mathrm{PhC}$ structure as it has to be optimized on the angular pattern of the photons that, in theory, could be extracted.

\section{A. Simulations of Scintillators With Plain Exit Surface}

The simulations of scintillators with plain exit surface were performed with the light ray tracing program LITRANI [9]. The system simulated with this program is given in Fig. 2 and is comprised of the following basic elements: a $2 \mathrm{~mm} \times 2 \mathrm{~mm}$ $\times 8 \mathrm{~mm}$ LuAP or LSO crystal with perfectly polished faces; a phototube (PMT) with a fused silica window and a photocathode with a quantum efficiency $Q_{\text {eff }}=1$; and a slice of optical glue between crystal and PMT.

The refractive index of LSO was approximated by using data measured at Caltech [10]. In the case of LuAP, a fixed value of 1.94, corresponding to the mean refractive index at the wavelength of maximum emission $\left(\lambda_{\max , \mathrm{LuAP}}=365 \mathrm{~nm}\right)$ [11], was used as no data are so far published on the ordinary and extraordinary refractive indices of this material and their wavelength dependence. However, assuming that a) the difference between $n_{\text {ord }}$ and $n_{\text {ext }}$ is small (i.e., $<0.05$ ), and b) both indices do not vary by more than \pm 0.02 over the whole wavelength range of emission (approx. from $330 \mathrm{~nm}$ to $410 \mathrm{~nm}$ ), the effect of this approximation on the results is expected to be small.

Approximate absorption lengths of both materials were determined by measuring the transmission of each type of crystal in air and re-calculating the absorption length $X_{0}$ by the formula

$$
\begin{aligned}
T & =\frac{t^{2} \cdot e^{-d / X_{0}}}{1-r^{2} \cdot e^{-2 \cdot d / X_{0}}}, \\
r & =\left|\frac{n(\lambda)-1}{n(\lambda)+1}\right|^{2}, \quad t=1-r
\end{aligned}
$$

where $r$ and $t$ are the reflectance and the transmittance and $d$ is the thickness of the material. Refractive index and absorption length of the glue and the PMT entry window were taken from the LITRANI database and from [12], respectively.

The side and end faces of the crystals were simulated with two different kinds of wrapping: a diffusive one (DuPont ${ }^{\mathrm{TM}}$ Tyvek $^{\circledR}$ ) and an ideally reflecting one. Between the crystal and the wrapping, a thin slice of air was introduced. In both cases, the diffusion coefficients and the real and imaginary parts of the refractive indices were taken from the LITRANI database.

In the center of each crystal, an internal light source radiating isotropically in all directions was placed to simulate the scintillating light. The emission spectra of each material were approximated by fitting absorption-corrected X-ray excited spectra of LSO and LuAP with a sum of two Gaussian curves. In both cases, the low-energy tails of the emission spectra were not taken into account.

\section{B. Simulations of Scintillators With Patterned Exit Surface}

The optical transmission of an exit surface with a PhC pattern was determined by means of a scattering matrix algorithm [13]. This formalism allows for the calculation the energy flow through a series of $N$ interfaces by expanding the electric and magnetic fields of the incoming, transmitted, and reflected waves into a Fourier series. The amplitudes of the partial waves are then connected by a scattering matrix $S$ :

$$
\left(\begin{array}{c}
\vec{A}_{0} \\
\vec{B}_{N}
\end{array}\right)=S \cdot\left(\begin{array}{c}
\vec{A}_{N} \\
\vec{B}_{0}
\end{array}\right) .
$$

The vectors $\vec{A}_{0}, \vec{B}_{N}, \vec{A}_{N}$ and $\vec{B}_{0}$ contain the in-plane field components of the incoming waves in positive and negative $z$-direction, the field components of the transmitted wave, and the field components of the reflected wave, respectively. Simulated were only cases with $\vec{B}_{N}=\overrightarrow{0}$, i.e., cases where the light was approaching the $\mathrm{PhC}$ slab only from the scintillator side (Fig. 3).

The model structure simulated with this method is sketched in Fig. 4. It consists of an LSO or LuAP substrate with a PhC 


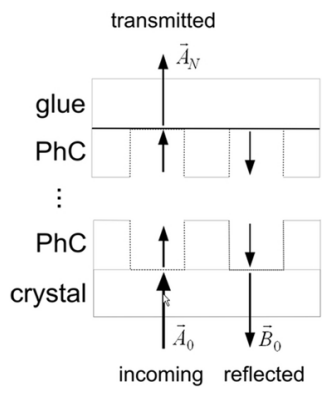

Fig. 3. System simulated with the scattering matrix algorithm. The fields of the reflected and transmitted waves are calculated from the fields of the incoming wave by the total scattering matrix of the system.

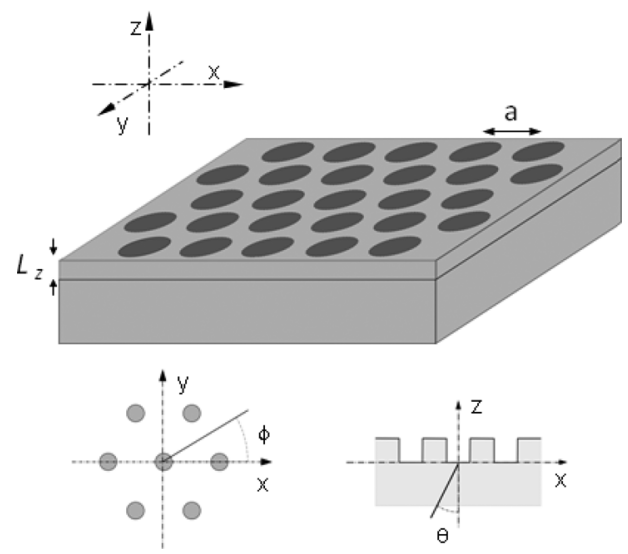

Fig. 4. Schematic structure of a PhC slab with a triangular pattern of air holes (lattice constant a) on top of a bulk material, as simulated with the scattering matrix algorithm. The electromagnetic wave is traveling in positive $z$ direction before it hits the $\mathrm{PhC}$. The thickness and lattice constant of the $\mathrm{PhC}$ are indicated in the plot. For reasons of clarity, the glue layer that follows the $\mathrm{PhC}$ in $z$ direction is not shown in this image.

consisting of a regular pattern of air holes in an LSO or LuAP matrix, and a layer of glue on top. The refractive index of the substrate and the glue was kept fixed, with $n_{\text {Glue }}=1.5, n_{\text {sub }}=$ 1.82 for LSO, and $n_{\text {sub }}=1.94$ for LuAP. The PhC was simulated as of square or triangular geometry with lattice constant a. Additionally, the hole radii $r$ and shapes were varied. Quasicrystalline lattices with higher symmetry than the triangular lattice were not simulated but are expected to lead to a further improvement of the light extraction efficiency due to the increased number of directions in which diffraction could take place [3].

In each run, between 85 and 151 plane waves were simulated to assess the principal optical properties of the simulated systems. Transmissions were determined for incident angles $\theta$ between $0^{\circ}$ (normal incidence) and $90^{\circ}$ and polar angles $\phi$ between $0^{\circ}$ and $45^{\circ}$ (square symmetry) and $0^{\circ}$ and $30^{\circ}$ (triangular symmetry). Two different polarization states of the wave were considered: for $S$ polarized waves, the electric field is oriented parallel to the $x-y$ plane, whereas for $P$ polarized waves, the magnetic field is parallel to the $x-y$ plane.

\section{RESULTS AND DISCUSSION}

\section{A. Simulations of Scintillators With Plain Exit Surface}

Fig. 5 shows the angular distribution of the photons arriving at the exit surface of a LuAP crystal versus the total number of

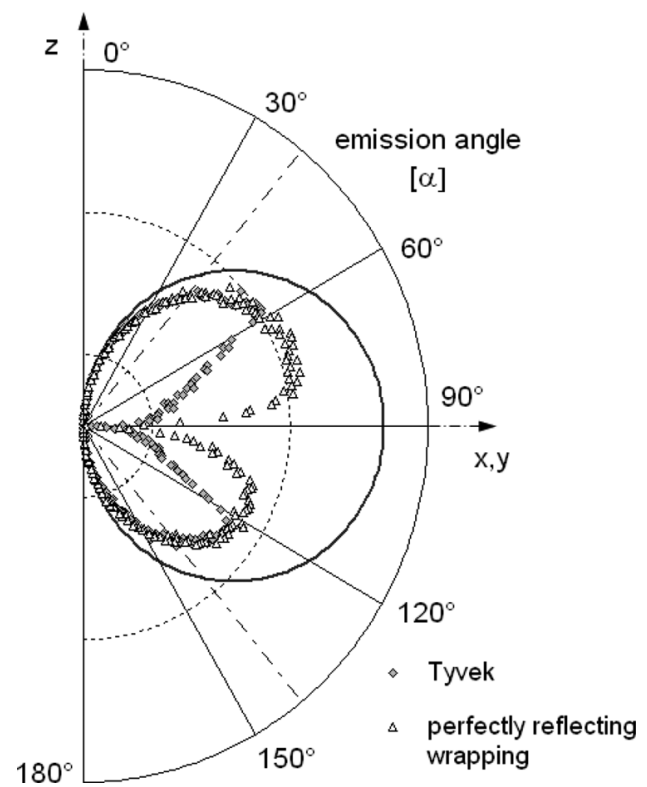

Fig. 5. Angular distribution of the photons that reach the exit surface of a LuAP crystal. The angle $\alpha$ corresponds to the angle between the $z$ axis and the $k$ vector of the photon at birth. Filled diamonds correspond to a crystal with Tyvek ${ }^{\circledR}$ wrapping around the outer faces and open triangles to a crystal wrapped with a perfectly reflecting material. The circle that envelops these two curves corresponds to the total number of photons that are generated per unit of angle.

photons generated per unit of angle. Diamonds correspond to a crystal with diffusing wrapping while triangles correspond to a crystal wrapped with an ideal reflector. As indicated in Fig. 2, the angle $\alpha$ is the angle between the wave vector $\vec{k}$ of the photon and the $z$ axis at the moment of emission.

This curve reveals some interesting details: First, it is apparent that the majority of photons emitted at angles close to the $x$ - $y$ plane (i.e., at an angle close to $90^{\circ}$ relative to the $z$ axis) does not reach the exit surface but instead, are absorbed. And second, the total number of photons that arrive at the exit surface greatly depends on the wrapping used to envelop the crystal: Whereas the curve is rather smooth for the ideal reflector, a significant drop can be observed in the case of a diffusive wrapping at emission angles between $60^{\circ}$ and $120^{\circ}$. This peculiar behavior is related to the fact that a photon travelling at such angles can exit the crystal through the lateral faces and will eventually hit the wrapping. In the case of a reflecting wrapping, the photon is simply reflected back and the angle relative to the $z$ axis is preserved when the photon re-enters the crystal. A diffusive wrapping, however, changes its angle in a random fashion, and a large fraction of the photons that would otherwise reach the exit surface will bounce back and forth between the side walls of the crystal with ever changing angles until they finally get absorbed.

Interestingly, this does not essentially mean that a much higher LEE is observed in the case where the crystal is wrapped with an ideal reflector. This is shown in Fig. 6, which plots the angular distribution of the arriving and extracted photons relative to the normal vector of the exit surface for both types of wrapping. On the other hand, this means that a large amount of photons is lost as their incident angle $\theta$ is larger than $\theta_{\text {tot }}$. Assessing these photons therefore holds strong potential to increase the LEE of the crystals. 

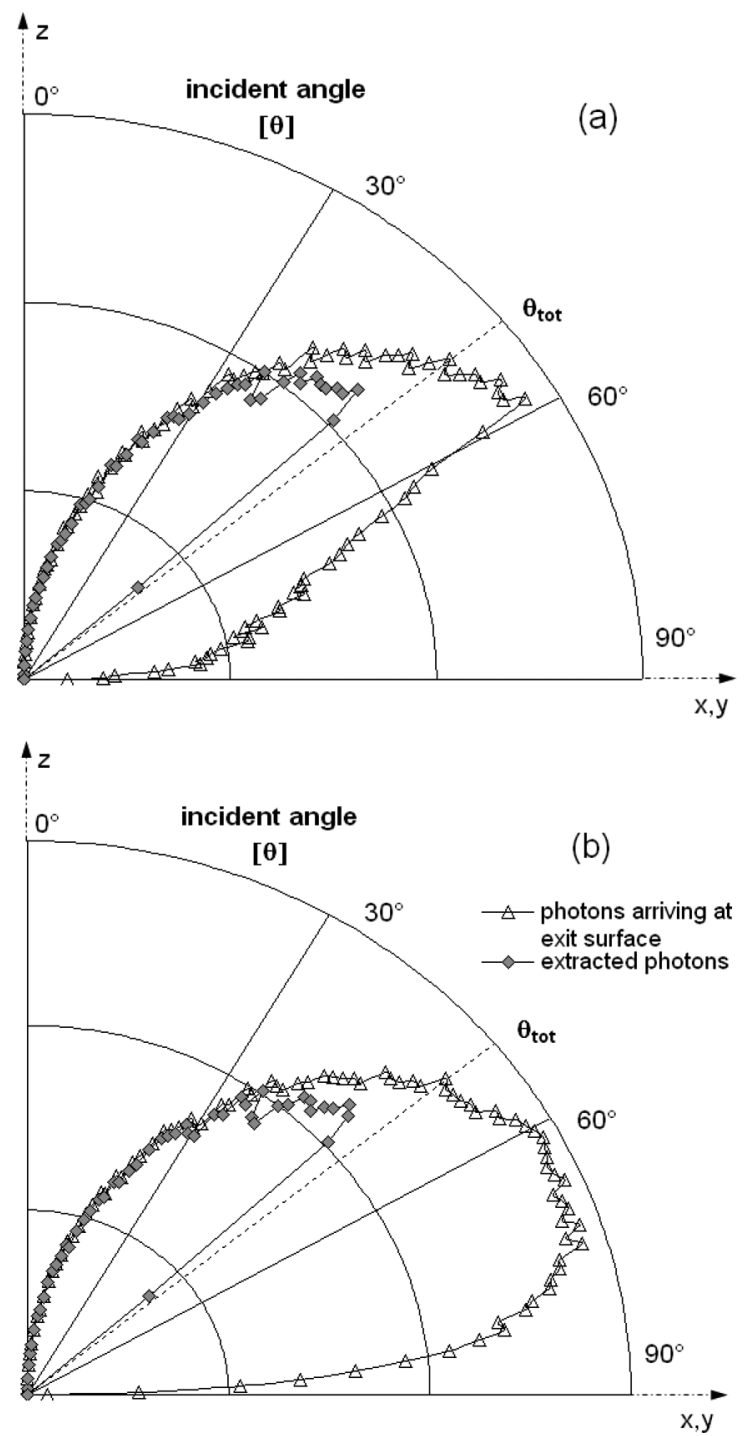

Fig. 6. Angular distribution of photons arriving at the exit surface and extracted photons for (a) Tyvek ${ }^{\circledR}$ and (b) perfectly reflecting wrapping in case of LuAP. The angle $\theta$ is defined as the angle between the k vector of the photon and the normal vector of the exit surface. Open triangles and filled diamonds correspond to the arriving and extracted photons, respectively. The dashed line indicates the angle of total reflection $\theta_{\text {tot }}$ at the interface crystal-glue for the wavelength of maximum emission. It is apparent that the number of extracted photons does not differ significantly for both wrappings.

\section{B. Simulations of Scintillators With Patterned Exit Surface}

Fig. 7 shows the transmission of an $\mathrm{S}$ polarized wave at normal incidence through the exit surface of the LuAP and LSO crystals in a case where the exit surface is patterned with a triangular PhC. Obviously, the resulting transmission curves are not smooth over the entire range of $a / \lambda$, but show pronounced dips and maxima. This is related to the contribution of different harmonics to the total transmission: the dips and maxima correspond to wavelengths where new harmonics are transmitted into the glue layer or reflected back into the substrate of the scintillator. It is also apparent that at this incident angle the total transmission is always slightly smaller than that of a crystal with plain exit surface.

A different behavior is observed when the photon is incident at the exit surface with an angle that is larger than $\theta_{\text {tot }}$ (Fig. 8). A

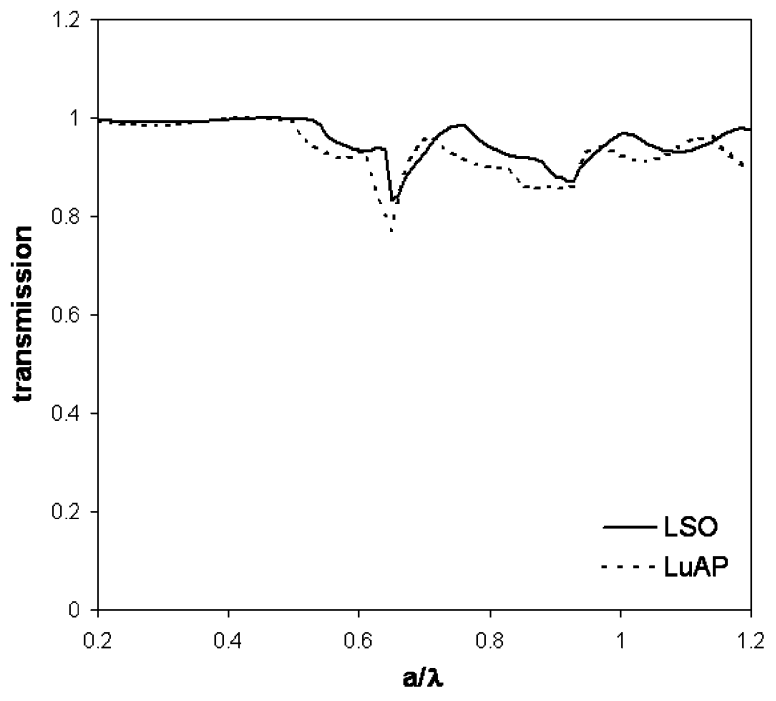

Fig. 7. Transmission of a scintillator-glue interface at normal incidence $(\theta=$ $0^{\circ}$ ) when the exit surface of the crystal is patterned with a triangular $\mathrm{PhC}$ (hole radius $r=0.29 a$ ). Solid line: LSO; dashed line: LuAP. The incoming wave is $S$ polarized and is incident onto the exit surface with a polar angle $\phi=0^{\circ}$, i.e., the magnetic field is orientated along $y$. The transmission of a plain exit surface is in both cases near 1 at this incident angle. 85 plane waves were used in the calculations.

surface without a PhC pattern reflects back all light that travels at such angles and the transmission is zero. If a $\mathrm{PhC}$ pattern is introduced, however, one or more harmonics can be extracted and the transmission can reach relatively high values under certain conditions. In other words, a PhC patterned exit surface allows for the extraction of a significant fraction of the photons that are normally lost by total internal reflection. By folding the average transmission per incident angle with the angular distribution of the photons arriving at the exit surface, it should be possible therefore to tune the structure of the $\mathrm{PhC}$ and the size of its elements so that the LEE of the crystal is optimized.

\section{CONCLUSIONS}

It is shown by means of simulations with the light ray tracing program LITRANI that in scintillators with high refractive index, a large amount of the photons produced in a particle event cannot be extracted due to total internal reflection at the exit surface. This problem can be partly overcome by machining a regular array of nanoholes into the exit surface of the crystal. By means of a scattering matrix algorithm, it is shown that the transmission of the exit surface with a $\mathrm{PhC}$ of lattice constant $\mathrm{a}$ is non-zero even for incident angles larger than the angle of total reflection and that it can reach high values for certain combinations of incident angle and $a / \lambda$. Folding the average transmission per incident angle with the angular distribution of the photons that arrive at the exit surface makes it possible to tune the structure of the $\mathrm{PhC}$ so that light extraction from the crystal is optimized.

\section{ACKNOWLEDGMENT}

The authors would like to thank M. Thiel and R. Nowotny from the Justus Liebig Universität, Giessen, Germany, for calling attention to the LSO refractive index measurements 


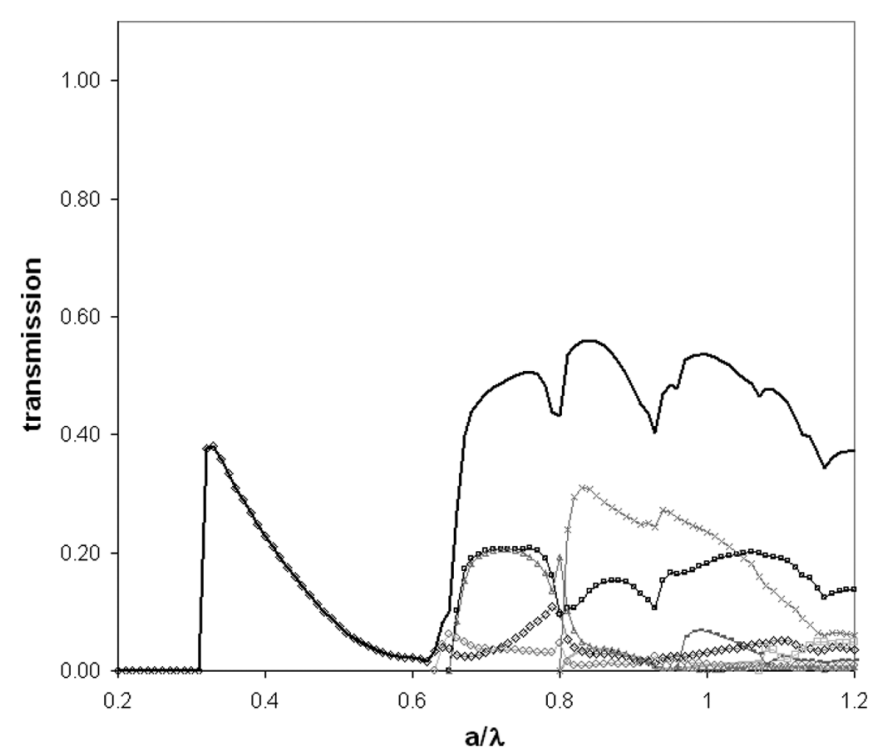

Fig. 8. Transmission of a LuAP crystal with a triangular $\mathrm{PhC}$ pattern at the exit surface (hole radius $r=0.29 a$ ) for an $S$-polarized photon with an incident angle $\theta=55^{\circ}$ and $\phi=0^{\circ}$. Several harmonics contribute to the total transmission (solid line). Note that a LuAP crystal with a plain exit surface has zero transmission at this incident angle. 85 plane waves were used in the calculation.

from Mao et al.; A. David from the Ecole Polytechnique, Palaiseau, France, for helping with building the scattering matrix formalism; and F. X. Gentit for his suggestions on LITRANI.

\section{REFERENCES}

[1] S. Fan, P. R. Villeneuve, J. D. Joannopoulos, and E. F. Schubert, "High extraction efficiency of spontaneous emission from slabs of photonic crystals," Phys. Rev. Lett., vol. 78, pp. 3294-3294, 1997.

[2] A. David et al., "Photonic-crystal GaN light-emitting diodes with tailored guided modes distribution," Appl. Phys. Lett., vol. , 88, pp. 061124-061124, 2006.

[3] A. David et al., "GaN light-emitting diodes with archimedean lattice photonic crystals," Appl. Phys. Lett., vol. 88, pp. 073510-073510, 2006.

[4] A. A. Erchak et al., "Enhanced coupling to vertical radiation using a two-dimensional photonic crystal in a semiconductor light-emitting diode," Appl. Phys. Lett., vol. 78, pp. 563-563, 2001.

[5] H. Y. Ryu, Y. H. Lee, R. L. Sellin, and D. Bimberg, "Over 30-fold enhancement of light extraction from free-standing photonic crystal slabs with InGaAs quantum dots at low temperature," Appl. Phys. Lett., vol. 79, pp. 3573-3573, 2001.

[6] M. Rattier et al., "Toward ultrahigh-efficiency aluminium oxide microcavity light-emitting diodes: Guided mode extraction by photonic crystals," IEEE J. Quantum Electron., vol. 8, pp. 238-238, 2002.

[7] Y. J. Lee et al., "A high-extraction-efficiency nanopatterned organic light-emitting diode," Appl. Phys. Lett., vol. 82, pp. 3779-3779, 2003.

[8] M. Zelsmann et al., "Seventy-fold enhancement of light extraction from a defectless photonic crystal made on silicon-on-insulator," Appl. Phys. Lett., vol. 83, pp. 2542-2542, 2003.

[9] F. X. Gentit, "Litrani: A general purpose Monte-Carlo program simulating light propagation in isotropic or anisotropic media," Nucl. Instrum. Methods Phys. Res. A, vol. A486, pp. 35, 2002-35, 2002.

[10] R. Mao, L. Zhang, and R. Y. Zhu, "Optical and scintillation properties of heavy crystal scintillators," in Proc. SCINT Conf., Winston-Salem, NC, Jun. 2007, CD-ROM.

[11] M. V. Korzhik, O. V. Misevich, and A. A. Fyodorov, "YA1O ${ }_{3}$ :Ce scintillators: Application for X- and soft $\gamma$-ray detection," Nucl. Instrum. Methods Phys. Res. B, vol. B72, pp. 499-499, 1992.

[12] E. D. Palik and E. J. Prucha, Handbook of Optical Constants of Solids. New York: Academic, 1998.

[13] S. G. Tikhodeev, A. L. Yablonskii, E. A. Muljarov, N. A. Gippius, and T. Ishihara, "Quasiguided modes and optical properties of photonic crystal slabs," Phys. Rev. B, vol. 66, pp. 045102-045102, 2002. 